\title{
ON RELATIVELY INVARIANT MEASURES
}

\author{
MARK MAHOWALD
}

1. Introduction. In this note we will discuss the question of the measurability of the multiplier function of a relatively invariant measure on a group. That is, for a group $G, \sigma$-ring $S$, and a measure $\mu$ defined on the sets of $S$, we assume: $E$ in $S, x$ in $G$ implies $x E$ is in $S$ and $\mu(x E)=\sigma(x) \mu(E)$ and study the measurability of the function $\sigma(x)$.

The problem was discussed by Halmos (1, p. 265), on locally compact groups and there the situation proved to be as nice as it could be, that is, if the measure is a non-trivial, relatively invariant Baire measure then the multiplier function is continuous. We prove two theorems for groups in which no topology is assumed. In the first theorem we assume a shearing condition and answer the question completely. The second theorem places a condition on the measure and weakens the shearing assumption. Its proof is complicated and occupies the major portion of this paper.

2. Definitions and Notation. We shall use the measure-theoretic notation and definitions of $(\mathbf{1})$ with these modifications and additions. All measures which are considered are complete.

2.1. A left-invariant ring, $R$, is a ring of subsets of a group, $G$ such that $E$ in $R$ implies $x E$ is in $R$ for all $x$ in $G$.

2.2. When we say a function, $f$, is $S$-measurable we mean that for $E$ in $S$ and $M$ a Borel set of the real line, $E \cap f^{-1}(M) \cap N(f)$ is in $S .(N(f)=$ $\{x: f(x) \neq 0\}$.)

2.3. $(G, S, \mu)$ will be a measure space such that $G$ is a group and $S$ is a left-invariant $\sigma$-ring of subsets.

2.4. If $E$ and $x E$ are measurable and $\mu(x E)=\sigma(x) \mu(E)$ and if $\mu$ is not identically equal to zero and is $\sigma$-finite then $\mu$ is called relatively invariant and will be denoted by $(\sigma) \mu$. Note that the definition of $\sigma(x)$ implies that $0<\sigma(x)<\infty$, all $x \in G, \sigma(x y)=\sigma(x) \sigma(y)=\sigma(y x), \sigma(e)=1, \sigma(x) \sigma\left(x^{-1}\right)=1$.

2.5. By $H(S)$ we shall mean the hereditary $\sigma$-ring generated by $S$.

2.6. In $\left(G, H(S),(\sigma) \mu^{*}\right)$ we shall define an outer measure integral denoted by $\delta^{*}(E)=\int{ }_{E} f(x) d \mu^{*}$, where $f$ is an arbitrary non-negative function on $G$ and

$$
\delta^{*}(E)=\lim _{n \rightarrow \infty} \sum_{i=1}^{n 2^{n}}(i-1) 2^{-n} \mu^{*}\left(E_{n i} \cap E\right)
$$

Received March 11, 1959. This paper is part of a thesis submitted to the University of Minnesota. The author is indebted to Professor Gelbaum for his help and guidance during the preparation of this paper. In addition, the author wishes to thank the referee for helpful suggestions, particularly in the proof of Theorem 2 . 
where $E_{n i}=\left\{x:(i-1) 2^{-n} \leqslant f(x)<i 2^{-n}\right.$ for $\left.i=1, \ldots, n 2^{n}\right\}$. Note that $0<\sigma(x)<\infty$ implies that if $\delta^{*}=\int{ }^{*} \sigma\left(x^{-1}\right) d \mu^{*}$, then $\delta^{*}(E)=0$ if and only if $\mu(E)=0$.

2.7. $(G, S)$ will be said to satisfy the shearing condition if the transformation from $G \times G$ to $G \times G$ defined by $\theta(x, y) \rightarrow \theta(x, x y)$ is a measurability preserving transformation, (carries $S \times S$ onto $S \times S$ ).

2.8. By weak shearing we shall mean that if $f(x)$ is $S$-measurable then $g(x, y)=f(x y)$ is $S \times S$-measurable.

2.9. By condition $A$ on a measure space we shall mean that the space is the union of a disjoint class $\mathscr{D}$ of measurable sets of finite measure with the property that every measurable set may be covered by countably many sets of $\mathscr{D}$ and a set of measure zero.

Remark. According to Halmos (1, p. 132) this implies that the RadonNikodym theorem is valid.

2.10. We say that $(G, S, \mu)$ is countably coverable if for every set $E$ of positive measure and any other measurable set $F$, there exist $x_{i}, i=1,2, \ldots$, such that $F-\cup x_{i} E$ has measure zero.

Remark. Lebesgue measure is countably coverable.

2.11. By a measure group we shall mean a measurable space $(G, S)$ such that $G$ is a group and $S$ is left-invariant and satisfies the shearing condition.

\section{Measurability theorems.}

THEOREM 1. Let $(G, S)$ be a measure group and let $(\sigma) \mu$ be a relatively invariant measure defined on $S$. Then $\sigma$ is $S$-measurable.

Proof. From the definition of shearing we have, for any subset $E$ of $G \times G$, $(\theta(E))_{x}=x E_{x}$. (See (1), p. 258.) Let $E=F \times F$, where $F$ is in $S$. By Fubini's theorem we have that

$$
\int \chi_{\theta(E)} d \mu(y)=\mu\left((\theta(E))_{x}\right)=\mu\left(x E_{x}\right)=\sigma(x) \mu(F) \chi_{F}
$$

is a measurable function of $x$. Therefore, $\sigma(x) \mu(F) \chi_{F}$ is measurable but $\mu(F)$ is a constant and $F$ is an arbitrary set in $S$; hence $\sigma(x)$ is $S$-measurable.

COROLLARY. In a measure group the existence of one non-trivial measure $(\sigma) \mu$ implies that any other non-trivial $\left(\sigma^{\prime}\right) \mu^{\prime}$ can be written as

$$
\mu^{\prime}(E)=K \int_{E} \sigma^{\prime} / \sigma d \mu .
$$

Proof. The theorem implies that both $\sigma$ and $\sigma^{\prime}$ are measurable. Let $\theta(E)=\int_{E} \sigma\left(x^{-1}\right) d \mu$ and $\theta^{\prime}(E)=\int_{E} \sigma^{\prime}\left(x^{-1}\right) d \mu^{\prime}$. Both $\theta$ and $\theta^{\prime}$ are invariant measures and $(G, S, \theta)$ and $\left(G, S, \theta^{\prime}\right)$ are measurable groups (see (1), page 257). Therefore Theorem $60: \mathrm{B}$ of (1) applies and shows that $K \theta=\theta^{\prime}$. Let 


$$
f_{n}=\sum_{m=1}^{M} a_{n m} \chi_{E_{n m}}
$$

be a sequence of simple functions monotonically converging to $\sigma^{\prime}$. Then

$$
\begin{aligned}
\mu^{\prime}(E)=\int_{E} \sigma^{\prime}(x) d \theta^{\prime}=\lim _{n \rightarrow \infty} \int_{E} f_{n} d \theta^{\prime} & \\
& =\lim _{n \rightarrow \infty} K \sum_{m=1}^{M} a_{n m} \int_{E_{n m} \cap_{E}} \sigma\left(x^{-1}\right) d \mu=K \int_{E}\left(\sigma^{\prime} / \sigma\right) d \mu .
\end{aligned}
$$

For Theorem 2 we shall need the following lemmas:

LEMma 1. For arbitrary non-negative function $f$ on $G, \delta^{*}$, the outer measure integral of $f$ in $\left(G, H,(S), \mu^{*}\right)$, is an outer measure on $H(S)$ and the $\sigma$-ring of $\mu^{*}$-measurable sets is contained in the $\sigma$-ring of $\delta^{*}$-measurable sets.

Proof. The fact that $\delta^{*}$ is an outer measure follows immediately from the definition. Let $E$ be $\mu^{*}$-measurable. Then for arbitrary $A \in H(S)$ we have

$$
\begin{aligned}
\delta^{*}(A) & =\lim _{n \rightarrow \infty} \sum_{i=1}^{n 2^{n}}(i-1) 2^{-n} \mu^{*}\left(A \cap E_{n i}\right) \\
& =\lim _{n \rightarrow \infty} \sum_{i=1}^{n 2^{n}}(i-1) 2^{-n}\left[\mu^{*}\left(A \cap E_{n i} \cap E\right)+\mu^{*}\left(A \cap E_{n i} \cap E^{\prime}\right)\right] \\
& =\delta^{*}(A \cap E)+\delta^{*}\left(A \cap E^{\prime}\right)
\end{aligned}
$$

This completes the proof of the lemma.

Lemma 2. If $\delta^{*}(E)=\int{ }_{E} f d \mu^{*}$, then $f$ is $R$-measurable, where $R$ is the collection of $\delta^{*}$-measurable sets.

Proof. It is sufficient to show $E_{N j}$ satisfies the Carathéodory criterion for all $A \in H(S)$. For $N$ and $j$ fixed and $n>N$, we have either $E_{n i} \cap E_{N j}=\phi$ or $E_{n i}$. Therefore, for arbitrary $A \in H(S)$ we have

$$
\begin{aligned}
\delta^{*}(A) & =\lim _{n \rightarrow \infty} \sum_{i=1}^{n 2^{-1}}(i-1) 2^{-n}\left(\mu^{*}\left(E_{N j} \cap A \cap E_{n i}\right)+\mu^{*}\left(E_{N j}^{\prime} \cap A \cap E_{n i}\right)\right) \\
& =\delta^{*}\left(A \cap E_{N_{j}}\right)+\delta^{*}\left(A \cap E_{N j}^{\prime}\right) .
\end{aligned}
$$

Lemma $3 . \delta^{*}(E)=\int{ }_{E} \sigma\left(x^{-1}\right) d \mu^{*}$ is an invariant outer measure on $H(S)$ and the restriction of $\delta^{*}$ to $S$ is an invariant measure on $S$.

The proof of this lemma is long and will be given in $\S 4$. We now have this

Theorem 2. Let $(G, S,(\sigma) \mu)$ satisfy condition $A$ and be countably coverable and suppose that there exists a set $E \in S$ such that $0<\delta^{*}(E)<\infty$, (with $\delta^{*}$ as in Lemma 3 ). Then there exists a $\sigma$-ring $R$ containing $S$ and a measure $(\sigma)$ $\mu$ on $R$ which is an extension of $\mu$ on $S$ such that $\sigma$ is $R$-measurable. If in addition $S$ satisfies the weak shearing condition then $\sigma(x)$ is $S$-measurable. 
Proof. By Lemma 3, $\delta^{*}$ restricted to $S$ is a measure. Since $\delta^{*}(E)=0$ if $\mu(E)=0, \delta^{*} \ll \mu$ and condition $A$ then implies the Radon-Nikodym theorem is valid. Let $f$ be the $R-N$ derivative. Let $E \in S$ be such that $0<\delta^{*}(E)<\infty$. Let $A$ be any set of $\mathscr{D}$. There exist $\left\{x_{i}\right\}, i=1,2, \ldots, 1$ such that $\cup x_{i} E \supset A$. Therefore, on $A, \delta$ is $\sigma$-finite. Hence $f$ can be chosen to be finite-valued on $A$, hence on $G$. On each subset $F$ of $A$ such that $\delta^{*}(F)<\infty$, we have,

$$
\delta^{*}(F)=\int_{F} f(y) d \mu=\delta^{*}(x F)=\int_{x F} f(y) d \mu=\sigma\left(x^{-1}\right) \int_{F} f\left(x^{-1} y\right) d \mu .
$$

Therefore, for each $x$

$$
f(y)=\sigma\left(x^{-1}\right) f\left(x^{-1} y\right), \quad[\mu] \text { in } y \text { for } y \text { in } A .
$$

Since the $A$ are disjoint and a countable union of them cover any measurable set to within a set of measure zero the formula is valid for all $x,[y]$, when $y$ is restricted to any measurable set.

(1) implies $\bar{\mu}=\int(f(x))^{-1} d \delta$ is a relatively invariant measure with $\delta$ as the multiplier function on the $\sigma$-ring of $\delta^{*}$ measurable sets $R$. Lemma 2 shows that $\sigma$ is $R$-measurable. Therefore, we have only to show that $\bar{\mu}$ is an extension of $\mu$. Using Theorem $B$, page 134 of (1), we have

$$
\int_{E}(f)^{-1} d \delta=\int_{E}(f)^{-1} f d \mu=\mu(E),
$$

for every $E$ in $S$. Therefore, $\bar{\mu}$ satisfies the theorem and this completes the proof of the first part of the theorem.

The weak shearing condition implies that $f(y) \sigma\left(x^{-1}\right)-f(x y)=g(x, y)$ is $R \times R$ measurable. On every set in $R \times R, g(x, y)$ is integrable and its integral will be zero by (1) and the Fubini theorem. Let $A$ be any set in $D$ with $\mu(A)>0$. Then $\delta^{*}(A)>0$ and $A$ contains a set of points of positive $\mu$-measure at which $0<f(y)<\infty$. Let $E$ be the subset of $A \times A$ for which $f(y) \sigma\left(x^{-1}\right)$ $-f(x y) \neq 0$. Then $\bar{\mu} \times \bar{\mu}(E)=0$. Therefore, for almost all $y$ in $E$,

$$
\frac{f(y)}{\sigma(x)}-f(x y)=0 \quad[\bar{\mu}] .
$$

If $A_{y}=\left\{x: f(y) \sigma^{-1}(x)-f(x y)=0\right\}, \bar{\mu}\left(A_{y}\right)=0$ for almost all $y$ in $A$ by the Fubini theorem. If $\bar{\mu}\left(A_{y}\right)=0$, then $\delta^{*}\left(A_{y}\right)=0$. Whence $\mu\left(A_{y}\right)=0$, using 2.6. Thus there exists $y \in E$ with $0<f(y)<\infty$ and such that $f(y) \sigma^{-1}(x)$ $-f(x y)=0$ for almost all $x$ in $A[\mu]$. The measurability of $f(x y)$ then implies that $\sigma(x)$ is measurable in $A$, and the definition of $A$ implies that $\sigma(x)$ is $S$-measurable.

4. Proof of Lemma 3. We shall prove a sequence of remarks which will lead to the lemma. 
REMARK 1. $\mu^{*}(x E)=\sigma(x) \mu^{*}(E)$ for all $E$ in $H(S)$.

Proof. This statement is an immediate consequence of the definition of an outer measure and the relative invariance of $\mu$.

In the following let $E$ be any set in $H(S)$ such that $\mu^{*}\left(E_{n i} \cap E\right)<\infty$ for all $n$ and $i \neq 1$.

From Remark 1 we have

$$
\begin{gathered}
\delta^{*}(E)=\lim _{n \rightarrow \infty} \sum_{i=1}^{n 2^{n}}(i-1) / 2^{n} \mu^{*}\left(E_{n i} \cap E\right)=\lim _{n \rightarrow \infty} \sum_{i=1}^{n 2^{n}}(i-1) / 2^{n} \sigma(y) . \\
\mu^{*}\left(y E_{n i} \cap y E\right) .
\end{gathered}
$$

Let $A(N, i)=\left\{j:(i-1) / 2^{n} \sigma(y)<(j-1) / 2^{N}<j / 2^{N}<i / 2^{n} \sigma(y)\right\}$ for $i=1, \ldots, n 2^{n}$. Note that $j$ in $A(N, i)$ implies $E_{N}, \subset y E_{n i}$ and that

$$
\bigcup_{j \epsilon A(N, i)} E_{N j} \subset \bigcup_{j \epsilon A(M, i)} E_{M j}
$$

if $N \leqslant M$.

In Remarks 2 and 3 we shall be concerned with a particular $i$ and fixed $n$ and $y$; hence we shall suppress the $i$ in the notation $A(N)$.

REMARK 2.

$$
y E_{n i}=\lim _{N \rightarrow \infty} \bigcup_{j \epsilon A(N)} E_{N j} \cup I
$$

where $I=\left\{x: \sigma(x)=2^{n} \sigma(y) /(i-1)\right\}$.

Proof. From the definition of $A$ we see that the right side is a subset of the left side. Let $z$ be a member of the left side. Then

$$
\sigma\left(z^{-1}\right)=(i-1)\left(2^{n} \sigma(y)\right)^{-1}
$$

or

$$
(i-1)\left(2^{n} \sigma(y)\right)^{-1}<\sigma\left(z^{-1}\right)<i\left(2^{n} \sigma(y)\right)^{-1} .
$$

The first case implies $z$ is in $I$. For the second case there exists an $M$ and $j \in A(M)$ such that

$$
(i-1) / 2^{n} \sigma(y) \leqslant(j-1) 2^{-M}<\sigma\left(z^{-1}\right)<j / 2^{M}<i / 2^{n} \sigma(y) .
$$

Therefore $z$ is in the union over $A(M)$ and $\cup_{j \epsilon A(N)} E_{N}$ is an increasing sequence of sets; hence the remark follows.

REMARK 3. Let $a>0$ be arbitrary; then, for any $E \in H(S)$ such that $\mu^{*}\left(E_{n i} \cap E\right)<\infty$, there exists an $M$ such that

$$
\mu^{*}\left(y E_{n i} \cap y E\right) \leqslant \sum_{j \epsilon^{\prime} M} \mu^{*}\left(E_{M j} \cap y E\right)+\mu^{*}(I \cap y E)+a 2^{-n} n^{-3} .
$$

Proof. From Remark 2 we have 


$$
\begin{aligned}
\mu^{*}\left(y E_{n i} \cap y E\right) & \leqslant \mu^{*}\left(\lim _{N \rightarrow \infty} \bigcup_{j \in A(N)} E_{N j} \cap y E\right)+\mu^{*}(I \cap y E) \\
& =\lim _{N \rightarrow \infty} \mu^{*}\left(\bigcup_{j \in A(N)} E_{N j} \cap y E\right)+\mu^{*}(I \cap y E) \\
& \leqslant \lim _{N \rightarrow \infty} \sum_{j \in A(N)} \mu^{*}\left(E_{N j} \cap y E\right)+\mu^{*}(I \cap y E)
\end{aligned}
$$

Since the left side is finite, there exists an $M$ such that the remark holds.

We can do this for each $i$ obtaining an $M_{i}$. If we are given $y$ and fix $n$ such that $\sigma(y) n>1$ and if we let $N_{0}=\max \left\{M_{i}, \log _{2} \sigma(y)+n\right\}$ then Remark 3 holds uniformly in $i$ for all $N \geqslant N_{0}$. In addition, since $1 / 2^{n} \sigma(y)>1 / 2^{N}$, there exists one distinct $j_{i}$ for each $i$ such that

$$
E_{N j_{i}} \supset I \text {. }
$$

We then can prove

Remark 4.

$$
\begin{aligned}
& \sum_{i=1}^{n 2^{n}}(i-1)\left(2^{n} \sigma(y)\right)^{-1} \mu^{*}\left(y E_{n i} \cap y E\right) \\
& \quad \leqslant \sum_{j=1}^{N 2^{N}}(j-1) 2^{-N} \mu^{*}\left(E_{N j} \cap y E\right)+\sum_{i=1}^{n 2^{n}} 2^{-N} \mu^{*}\left(E_{N j i} \cap y E\right)+a
\end{aligned}
$$

for all $N \geqslant N_{0}$.

Proof. We shall call the left side of the inequality $K_{n}$. Then, from Remark 3 and the definition of $A(N, i)$, we have

$$
\begin{aligned}
& K_{n} \leqslant \sum_{i=1}^{n 2^{n}}(i-1) / 2^{n} \sigma(y)\left[\sum_{j \in A(N, i)} \mu^{*}\left(E_{N j} \cap y E\right)+\mu^{*}(I \cap y E)\right]+\sum_{i=1}^{n 2^{n}} a(i-1) / n^{3} 2^{2 n} \sigma(y) \\
& \leqslant \sum_{i=1}^{n 2^{2 n}} \sum_{j \in A(N, i)}\left[(j-1) 2^{-N}\right] \mu^{*}\left(E_{N j} \cap y E\right) \\
&+\sum_{i=1}^{n 2^{n}}(i-1)\left(2^{n} \sigma(y)\right)^{-1} \mu^{*}(y E \cap I)+a .
\end{aligned}
$$

Since there exists one $j_{i}$ for each $i$, we have for all $N \geqslant N_{0}$

$$
\begin{aligned}
K_{n} \leqslant \sum_{j=1}^{N 2^{N}}(j-1) 2^{-N} \mu^{*}\left(E_{N j} \cap y E\right) & \\
& +a+\sum_{i=1}^{n 2^{n}}\left[(i-1)\left(2^{n} \sigma(y)\right)^{-1}-\left(j_{j}-1\right) 2^{-N}\right] \mu^{*}\left(E_{N j_{i}} \cap y E\right) .
\end{aligned}
$$

Since $(i-1)\left(2^{n} \sigma(y)\right)^{-1}-\left(j_{i}-1\right) 2^{-N} \leqslant 2^{-N}$, the remark follows.

Remark 5. The lemma is true if $\mu^{*}(E)<\infty$. 
Proof. If $\mu^{*}(E)=0$ we are finished. Therefore we shall assume that $0<\mu^{*}(E)<\infty$. Then from Remark 4 and the monotonicity of the outer measure, we have

$$
K_{n} \leqslant a+\delta^{*}(y E)+\left(n 2^{n} / 2^{N}\right) \mu^{*}(y E) .
$$

Letting $N \rightarrow \infty$ we have $K_{n} \leqslant a+\delta^{*}(y E)$. This is true for all $n$ from some point on; therefore, $\delta^{*}(E) \leqslant a+\delta^{*}(y E)$. Since $a$ is arbitrary we have $\delta^{*}(E) \leqslant \delta^{*}(y E)$. Applying this inequality to the set $y E$ and $y^{-1}$ we conclude that $\delta^{*}(E) \geqslant \delta^{*}(y E)$ and the remark follows.

REMARK 6. If $\mu^{*}(E)=\infty$, then the lemma is true if there exists a $K$ such that $\mu^{*}\left(y E_{n i} \cap y E\right)<2^{n} K$ for all $n$ and $i \neq 1$.

Proof. Since $1 / 2^{N}<1 / 2^{n} \sigma(y)$, we have

$$
E_{N j_{i}} \subset y E_{n i} \cup y E_{n, i-1} \text {. }
$$

Then from Remark 4 we have

$$
\begin{aligned}
K_{n} & \leqslant \delta^{*}(y E)+\sum_{i=1}^{n 2^{n}} 2^{-N}\left[\mu^{*}\left(y E_{n i} \cap y E\right)+\mu^{*}\left(y E_{n, i-1} \cap y E\right)\right]+a \\
& \leqslant \delta^{*}(y E)+22^{n} K n 2^{n} 2^{-N}+a .
\end{aligned}
$$

The remark now follows as in Remark 5 .

REMARK 7. If $\mu^{*}(E)=\infty$ and there does not exist a $K$ as in remark 6 , then the lemma is true.

Proof. Let $K^{\prime}$ be given. Then there exists an $n$ and $i_{0} \neq 1$ such that

$$
\mu^{*}\left(y E_{n i_{0}} \cap y E\right)=\sigma(y) \mu^{*}\left(E_{n i_{0}} \cap E\right)>2^{n} K^{\prime} \sigma(y) .
$$

This implies

$$
\delta^{*}(E) \geqslant \sum_{i=1}^{n 2^{n}}(i-1) 2^{-n} \mu^{*}\left(E_{n i} \cap E\right)>K^{\prime} .
$$

Hence $\delta^{*}(E)=\infty$. The result follows as in Remark 5 .

This completes the proof of the Lemma. The case which was excluded just before Remark 2, that is, $E$ such that $\mu^{*}\left(E_{n i} \cap E\right)=\infty$ for some $n$ and $i \neq 1$, is clearly covered in Remark 7 .

\section{REFERENCE}

1. P. R. Halmos, Measure theory (New York: Van Nostrand Co., Inc.).

Xavier University

Cincinnati, Ohio 